\title{
Factores implicados en la adherencia terapéutica en pacientes con fibromialgia
}

\author{
Mª Dolores Ruiz-Fernández ${ }^{1}$, Dolores Hernández-Martínez², Antonia \\ Fernández-Leyva ${ }^{1}$ y María López-Cano ${ }^{1}$ \\ ${ }^{1}$ SAS, Distrito Sanitario Almería (España); ${ }^{2}$ SAS, Distrito \\ Sanitario Poniente (España)
}

\begin{abstract}
La fibromialgia es una enfermedad crónica que requiere de un tratamiento farmacológico complejo y múltiple. Con el objetivo de estudiar el grado de adherencia terapéutica en esta enfermedad al tratamiento farmacológico prescrito y, conocer cómo influyen una serie de variables como son la edad, la salud percibida, el número de fármacos y la dificultad en la toma de medicación, se realizó un estudio descriptivo en 50 pacientes diagnosticados de fibromialgia. Los resultados nos muestran como la mayoría de los pacientes están polimedicados y un porcentaje amplio presenta mala adhesión al tratamiento, siendo en estos pacientes la puntuación media en salud percibida significativamente superior en problemas crónicos, con respecto a los que tienen una buena adherencia. El análisis de regresión logística multivariante nos indica un riesgo elevado de no adherencia en los pacientes que están polimedicados, tienen 60 o más años y presentan problemas mentales crónicos, aunque la asociación solo es significativa para los problemas crónicos. Por tanto, factores como el número de fármacos, la edad y la percepción que tienen sobre su salud, son variables a considerar en el grado de cumplimiento terapéutico en estos pacientes.
\end{abstract}

Palabras clave: Adherencia terapéutica, fibromialgia, tratamiento farmacológico, edad, salud percibida.

Factors involved in adherence to therapy in fibromyalgia patients. Fibromyalgia is a chronic disease that it needs of a complex and multiple drug therapy. With the aim to study the degree of adherence to therapy in this disease to the pharmacological treatment prescribed and, to know how they influence a series of variables as they are the age, the perceived health, the number of drugs and the difficulty in taking medication, a descriptive study was realized in 50 patients diagnosed of fibromyalgia. The results show us as the most patients are multiple medication and a large percentage present bad adherence to treatment, being in these patients the mean perceived health score significantly higher in chronic problems, compared to those with they have a good adherence. The multivariate logistic regression analysis indicates a high risk of non-adherence in patients who they are multiple medication, they are 60 or older and they have chronic mental problems, although the association only is significant for chronic problems. Therefore, factors as such the number of drugs, the age and the perception that they have about their health, are variables to take into consideration in the degree of adherence to therapy in these patients.

Keywords: Adherence to therapy, fibromyalgia, pharmacological treatment, age, perceived health.

Correspondencia: $\mathrm{M}^{\mathrm{a}}$ Dolores Ruiz Fernández. U.G.C. Cruz de Caravaca. Distrito Sanitario Almería. Servicio Andaluz de Salud. C/ Quinta Avenida, s/n. C.P. 04008. Almería (España). E-mail: mariad.ruiz.fernandez.sspa@juntadeandalucia.es 
La OMS (2004) ha definido la adherencia terapéutica como "el grado en que el comportamiento de una persona para tomar medicamentos, seguir un régimen alimentario y realizar cambios en su estilo de vida, se corresponde con las recomendaciones acordadas por un profesional sanitario". Una mala adherencia al tratamiento prescrito, repercute no solo en el empeoramiento de la enfermedad, si no que produce una mayor mortalidad y un aumento de los costos sanitarios y sociales (Jimmy y Jimmy, 2011). Por tanto, es un problema relevante en la práctica clínica por su frecuencia, así como por la repercusión que produce, lo que implica que su abordaje sea complejo y requiera de múltiples intervenciones (Orueta, 2005; Van Dulmen et al., 2007). Entre los elementos prioritarios para conseguir una buena adherencia al tratamiento se han estudiado una buena comunicación entre los pacientes y los profesionales sanitarios, junto con una intervención adaptada a las necesidades de los individuos (Butow y Sharpe, 2013).

En una clasificación acerca de los factores que pueden influir en el cumplimiento del régimen terapéutico prescrito, se han identificado cuatro bloques principales. En primer lugar, aquellos que tienen que ver con la relación clínica entre el paciente y el profesional sanitario, los que están relacionados con el tratamiento indicado, los que dependen de las características de la enfermedad y por último, los relacionados con las dimensiones psicosociales de la persona (Ginarte, 2001). Concretamente, entre las variables que están derivadas de las características de la enfermedad y más aún, en las patologías crónicas, como la hipertensión o la hipercolesterolemia, en las cuales el tratamiento farmacológico es continuo y periódico, se ha investigado que la adherencia al tratamiento es muy baja (Dimatteo, Giordani, Lepper, y Croghan, 2002).

La fibromialgia es una enfermedad crónica que está caracterizada principalmente por un dolor continuo, no inflamatorio y por un aumento de sensibilidad al tacto que afecta a las partes blandes del aparato locomotor, repercutiendo en la esfera biológica, psicológica y social de los pacientes (Villanueva et al., 2004). El principal síntoma es el dolor crónico, pero también es característico de la enfermedad el componente psicológico, con síntomas como la ansiedad y depresión, que hace que los afectados tengan un perfil psicológico característico (Pérez-Pareja et al., 2004; Ubago, Ruíz, Bermejo, Labry, y Plazaola, 2005). La prevalencia de fibromialgia es mayor en las mujeres que en los hombres e incluso su sintomatología difiere en función del género, causa situaciones de incapacidad laboral y repercute de forma considerable en el entorno familiar (Ubago, Ruiz, Bermejo, Labry, y Plazaola, 2005; Miró, Diener, Martínez, Sánchez, y Valenza, 2012).

El tratamiento farmacológico es complejo y muy variado. Entre los fármacos más utilizados en la enfermedad destacamos los analgésicos, antidepresivos, antiinflamatorios y anticonvulsionantes (Redondo, 2011). Sin embargo, no existen 
estudios que demuestren la eficacia de una pauta de tratamiento concreto para paliar la sintomatología, lo que implica que se estén utilizando otro tipo de terapias alternativas de forma coadyuvante con la medicación (Nishishinya, Rivera, Alegre, y Pereda, 2006), como pueden ser técnicas psicológicas como el midnfulness, con muy buenos resultados (Amutio, Franco, Pérez-Fuentes, Gázquez, y Mercader, 2015).

En la fibromialgia al igual que en otras enfermedades crónicas, en la que los pacientes están sometidos a un tratamiento complejo y variable de medicamentos, se aprecia un grado de incumplimiento terapéutico considerable. Entre los factores implicados, se encuentran las características individuales de los pacientes y de la enfermedad y en mayor medida, aquellos que tienen que ver con la relación terapéutica (Sewitch et al., 2004) y con problemas psicológicos (Dobkin y Sewitch, 2006). Concretamente, en un estudio realizado para valorar el grado de adherencia a un fármaco específico en fibromialgia se ha comprobado que factores como la edad o la toma de antidepresivos previos, estaban asociados con una alta adherencia terapéutica (Cui, Zhao, Novick, y Faries, 2012).

El objetivo de nuestro estudio consiste en analizar el grado de adhesión terapéutica al tratamiento farmacológico en pacientes con fibromialgia y conocer cómo influyen variables como la edad, la salud percibida, el número de fármacos y la dificultad en la toma de medicación, en el cumplimiento del tratamiento prescrito.

\section{MÉTODO}

\section{Participantes}

La muestra estaba compuesta por 52 mujeres diagnosticadas de fibromialgia, pertenecientes a la asociación de fibromialgia "AFIAL", en la provincia de Almería. La edad media de los participantes del estudio era de 56.83 (DT=7.03), siendo la edad mínima de 35 y la edad máxima de 69 años. Al categorizar la variable edad, tal y como puede observarse en la tabla 1 , el $53,8 \%$ de la muestra tenían una edad comprendida entre 51 y 60 años, el $30.8 \%$ de la muestra correspondía a más de 60 años y un $15.4 \%$ menos de 50 años. En lo referente al estado civil, un $59.6 \%$ de las mujeres estaban casadas, seguidas de los $28.8 \%$ que eran solteras, un $9.6 \%$ separadas y el resto viudas.

Tabla 1. Distribuciones de frecuencias: género, edad y estado civil

\begin{tabular}{cccccccccc}
\hline & \multicolumn{2}{c}{ Género } & \multicolumn{3}{c}{ Edad } & \multicolumn{4}{c}{ Estado civil } \\
\cline { 2 - 10 } & Hombres & Mujeres & 50 o menos & $51-60$ & 61 o más & Casado/a & Viudo/a & Soltero/a & Separado/a \\
\hline $\mathrm{N}$ & 0 & 52 & 8 & 28 & 16 & 31 & 1 & 15 & 5 \\
\hline$\%$ & $0 \%$ & $100 \%$ & $15.4 \%$ & $53.8 \%$ & $30.8 \%$ & $59.6 \%$ & $1.9 \%$ & $28.8 \%$ & $9.6 \%$ \\
\hline
\end{tabular}




\section{Instrumentos}

Para valorar la adherencia al tratamiento farmacológico se ha utilizado el test de Morisky-Green (Morisky, Green, y Levine, 1986), validado para la población española en distintas enfermedades crónicas, entre ellas la hipertensión (Val, Amorós, Martínez, Fernández, y León, 1992). Se trata de un cuestionario administrado mediante entrevista que consta de cuatro preguntas con respuestas dicotómicas ( $\mathrm{Si} / \mathrm{No}$ ) y que valora el grado de cumplimiento con respecto al tratamiento prescrito, proporcionando información sobre las causas del mismo. Se considera que el paciente es un buen cumplidor del tratamiento cuando responde a las preguntas de $\mathrm{No} / \mathrm{Si} / \mathrm{No} / \mathrm{No}$, por el contrario no está tomando bien la medicación según las indicaciones prescritas.

Otro instrumento utilizado, ha sido el cuestionario de Salud General de Goldberg (CHQ-28) (Lobo, Pérez-Echeverría, y Artal, 1986) que mide la salud percibida por el individuo y el bienestar emocional, en general. Su objetivo no es diagnosticar problemas psicológicos, pero si detecta trastornos psicológicos de reciente aparición y crónicos. Consta de 28 ítems y se divide en cuatro subescalas, de siete ítems cada una, que miden diferentes dimensiones: síntomas somáticos, ansiedad e insomnio, disfunción social y depresión grave. La fiabilidad oscila entre 0.82 y 0.93 .

\section{Procedimiento}

La selección de la muestra se hizo de forma aleatoria, entre los miembros de la asociación de pacientes con fibromialgia de Almería "AFIAL" y que acudieron a la implementación de un taller sociosanitario impartido por la unidad de gestión clínica de Plaza de Toros, en el Distrito Sanitario Almería, al encontrarse la sede de la asociación ubicada en esta zona.

Previamente, a la recogida de datos y administración de los cuestionarios correspondientes, se les solicitó el consentimiento informado a los asistentes, preservando en todo momento la confidencialidad y e intimidad, según la ley de protección de datos y las normas éticas de Helsinki, en cuanto a los derechos de los individuos en cualquier investigación médica.

\section{Diseño y Análisis de Datos}

Estudio descriptivo de corte transversal. En el estudio descriptivo de las variables cualitativas, se han utilizado distribuciones de frecuencias, tablas de contingencia y la prueba de Chi Cuadrado. En cambio, para las variables cuantitativas se han utilizado medidas de tendencia central y la prueba de $t$ de Student para muestras independientes. Como variable respuesta hemos tomado la adhesión al tratamiento (variable categórica) y como variables predictoras, la edad, la salud percibida, el número de fármacos y la dificultad en la toma de medicación. En un primer lugar, hemos realizado un análisis de regresión logística binaria con cada una de las variables 
predictores, calculando sus correspondientes razones de productos cruzados u odds ratios y sus respectivos intervalos de confianza. Posteriormente, se ha construido un modelo con todas la variables predictoras y se ha realizado un análisis de regresión logística multivariante, con el método de razones de máxima verosimilitud y el estadístico Wald, calculando las odds ratios ajustadas y sus intervalos de confianza correspondientes al $95 \%$.

Para el análisis de los datos se ha utilizado el programa estadístico SPSS, versión 22.

\section{RESULTADOS}

La siguiente tabla nos muestra la adherencia al tratamiento farmacológico, medida mediante el cuestionario de Morisky y su relación, con la prescripción de más de cinco fármacos diarios (polimedicación) y la dificultad en la toma de la medicación (Tabla 2). Como puede observarse, en un $32.7 \%$ de la muestra se observa una mala adherencia al tratamiento farmacológico, con respecto al $67.3 \%$ que si tiene una buena adherencia. La mayoría de las mujeres son polimedicadas y no presentan dificultad para tomar la medicación, tanto en las mujeres que tienen una buena como una mala adherencia al tratamiento. Sin embargo, estas diferencias observadas entre la polimedicación y la dificultad para tomar la medicación prescrita en función de la adherencia terapéutica, no son significativas estadísticamente.

Tabla 2. Adherencia al tratamiento, polimedicación y dificultad en la toma de medicación. Descriptivos

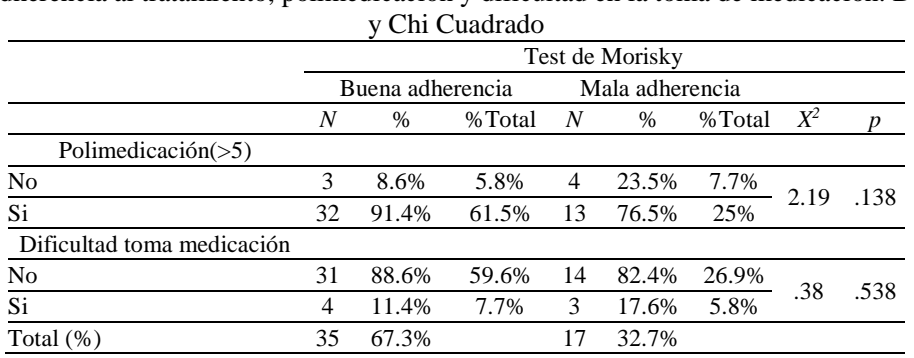

La salud percibida media, tanto para los problemas agudos como para los problemas crónicos, es mayor en aquellas mujeres que tienen una mala adherencia al tratamiento. La $t$ de Student para muestras independientes, nos muestra que la salud percibida para los problemas crónicos es significativamente superior en el grupo de mala adherencia, con respecto al de buena adherencia, medido mediante el cuestionario de Morisky (Tabla 3). 
Tabla 3. Adherencia al tratamiento y salud percibida (GHQ-28). Descriptivos y $t$ de Student

\begin{tabular}{lcccccccc}
\hline & \multicolumn{7}{c}{ Test de Morisky } \\
\cline { 2 - 9 } & Buena adherencia & \multicolumn{2}{c}{ Mala adherencia } \\
\hline GHQ-28 (p. agudos) & 35 & 14.29 & 4.73 & 17 & 15.94 & 6.05 & 1.07 & .286 \\
\hline GHQ-28 (p. crónicos) & 35 & 21.71 & 3.83 & 17 & 24.71 & 2.93 & 2.83 & $.007^{*}$ \\
\hline$* p<.05$ & & & & & & & &
\end{tabular}

El análisis de regresión logística, nos muestra la relación entre la adherencia al tratamiento farmacológico y las variables predictoras como la edad, el tomar más de cinco fármacos al día (polimedicación), la dificultad en la toma y la salud percibida, en los procesos agudos y los crónicos (Tabla 4). El análisis de regresión binario, nos indica como en las mujeres de 51 a 60 años, el riesgo de no adherencia a la medicación prescrita es de 2.6 veces más, en comparación con las de menor edad (50 o menos años). En las mujeres, el riesgo de no tener un buen cumplimiento en el tratamiento es de 3.28 veces más en las polimedicadas, con respecto a las no polimedicadas. Con respecto a la salud percibida, el riesgo para tener una mala adherencia al tratamiento es mayor para los procesos crónicos que para los agudos. En concreto, para los procesos crónicos el riesgo es de un $27 \%$, al compararlo con otra mujer que tiene una puntuación inferior, siendo además significativa su asociación.

Tabla 4. Análisis de regresión logística: Odds ratio (OR) e intervalos de confianza

\begin{tabular}{|c|c|c|c|c|c|c|}
\hline & OR cruda & IC* & $p$ & OR ajustada & $\mathrm{IC}^{*}$ & $p$ \\
\hline \multicolumn{7}{|l|}{ Edad } \\
\hline 50 o menos & 1 & & & 1 & & \\
\hline $51-60$ & 2.60 & $.38-17.45$ & .325 & 1.90 & $.21-17.13$ & .563 \\
\hline 61 o más & 2.08 & $.64-12.15$ & .168 & 2.82 & $.50-15.91$ & .243 \\
\hline GHQ-28 (p. agudos) & 1.06 & $.95-1.19$ & .282 & .89 & $.74-1.07$ & .228 \\
\hline GHQ-28 (p. crónicos) & 1.27 & $1.05-1.53$ & $.011^{* *}$ & 1.38 & $1.05-1.81$ & $.017 * *$ \\
\hline \multicolumn{7}{|c|}{ Polimedicación $(<5)$} \\
\hline No & 1 & & & 1 & & \\
\hline $\mathrm{Si}$ & 3.28 & $.64-16.74$ & .153 & 3.22 & $.49-21.08$ & .221 \\
\hline \multicolumn{7}{|c|}{ Dificultad toma medicación } \\
\hline No & 1 & & & 1 & & \\
\hline$\overline{\mathrm{Si}}$ & .602 & $.11-3.05$ & .541 & .43 & $.06-2.83$ & .383 \\
\hline
\end{tabular}

En el análisis de regresión multivariante ajustado al resto de variables del modelo, el riesgo de incumplimiento aumenta en las mujeres de más de 61 años con respecto, a las de menor edad (50 o menos años). Es decir, el riesgo de no adherencia en la mujeres de más de 61 años es 2.86 veces más con respecto a las de 50 o menos edad. Por el contrario, la odds ratio para la salud percibida en problemas agudos disminuye al ajustar el modelo $(\mathrm{OR}=.89)$. Sin embargo, aumenta significativamente para los problemas crónicos, es decir, el riesgo de tener una mala adherencia al tratamiento en las mujeres es del $38 \%$, en comparación con una mujer que tiene una puntuación en salud 
percibida menor en una unidad, a igualdad del resto de variables analizadas. En cuanto a la variable polimedicación el riesgo se mantiene elevado, siendo el riesgo de no adherencia 3.22 veces más elevado en las mujeres polimedicadas, tomando como referencia a las no polimedicadas (Tabla 4).

\section{DISCUSIÓN Y CONCLUSIONES}

Como se ha podido observar, todos los participantes del estudio son mujeres debido a que es una enfermedad prevalente en el sexo femenino, con un porcentaje muy pequeño de hombres diagnosticados con la enfermedad (Ubago et al., 2005; Miró et al., 2012). El tratamiento para paliar los síntomas de la enfermedad es complejo e incluye una gran variedad de fármacos, con distintos mecanismos de actuación. En nuestro estudio al igual que en otras investigaciones, la mayor parte de los pacientes están tomando más de cinco fármacos diarios (Redondo, 2011) y un porcentaje elevado, presenta una mala adherencia al tratamiento farmacológico indicado (Sewitch et al., 2004; Dobkin y Sewitch, 2006).

Se ha comprobado que uno de los principales factores que inducen al consumo de fármacos en atención primaria, en pacientes los pacientes diagnosticados de fibromialgia, es la salud que perciben y no tanto, el dolor crónico que padecen (Boyer, López-Roig, Calatayud, y Gelabert, 2010). Este aspecto es relevante en nuestra investigación, observándose como las mujeres describen que su salud, tanto para problemas agudos como para crónicos, es mala en ambos grupos de adherencia terapéutica, siendo más acentuada cuando existe incumplimiento al tratamiento y está relacionada, con problemas psicológicos crónicos. No debemos obviar que en la fibromialgia, existe un gran componente psicológico, característico de la enfermedad y que cursa principalmente con trastornos de ansiedad y depresión (Pérez-Pareja et al., 2004; Ubago et al., 2005).

La edad es otro factor relacionado con las características del paciente y que va a provocar una mala adherencia al tratamiento farmacológico, al igual que otros estudios en los que se ha tenido en cuenta esta variable, relacionándola con alto grado de cumplimiento terapéutico, junto con otras variables (Cui et al., 2012). En nuestra investigación las mujeres más longevas son las que tienen más riesgo de tener una mala adherencia terapéutica, en comparación con las mujeres más jóvenes.

A modo de conclusión, no debemos obviar que la fibromialgia es una enfermedad crónica reumatológica que requiere de un tratamiento continuado y prolongado de fármacos para paliar la sintomatología característica de la enfermedad. Por esta razón, una buena adherencia al tratamiento prescito, es fundamental para mejorar la calidad de vida de los pacientes afectados y en consecuencia, evitar o reducir 
todas las repercusiones que provoca esta enfermedad en el ámbito personal, social y laboral.

\section{REFERENCIAS}

Amutio, A., Franco, C., Pérez-Fuentes, M.C., Gázquez, J.J., y Mercader, I. (2015) Mindfulness training for reducing anger, anxiety, and depression in fibromyalgia patients. Frontiers in Psychology, 5, 1572. doi: 10.3389/fpsyg.2014.01572.

Boyer, A.L., López-Roig, S., Calatayud, N.P., y Gelabert, M.M.A. (2010). Predictores de la utilización de recursos sanitarios en la fibromialgia. Psicothema, 22(4), 549-555.

Butow, P., y Sharpe, L. (2013). The impact of communication on adherence in pain management. Pain ${ }^{\circledR}, 154$, S101-S107.

Cui, Z., Zhao, Y., Novick, D., y Faries, D. (2012). Predictors of duloxetine adherence and persistence in patients with fibromyalgia. Journal of Pain Research, 5, 193-201. doi:10.2147/JPR.S31800 [doi].

Dobkin, P.L., Sita, A., y Sewitch, M.J. (2006). Predictors of adherence to treatment in women with fibromyalgia. The Clinical Journal of Pain, 22(3), 286-294.

Dimatteo, M.R., Giordani, P.J., Lepper, H.S., y Croghan, T.W. (2002). Patient adherence and medical treatment outcomes: A meta-analysis. Medical Care, 40(9), 794-811.

Ginarte, Y. (2001). La adherencia terapéutica. Revista Cubana de Medicina General Integral, $17(5), 502-505$.

Jimmy, B., y Jimmy, J. (2011). Patient medication adherence: Measures in daily practice. Oman Medical Journal, 26(3), 155-159. doi:10.5001/omj.2011.38 [doi].

Lobo, A., Pérez-Echeverría, M.J., y Artal, J. (1986). Validity of the scaled version of the General Health Questionnaire (GHQ-28) in a Spanish population. Psychological Medicine, 16, 135-140.

Miró, E., Diener, F.N., Martínez, M.P., Sánchez, A.I., y Valenza, M.C. (2012). La fibromialgia en hombres y mujeres: Comparación de los principales síntomas clínicos. Psicothema, 24(1), 10-15.

Morisky, D.E., Green, L.W., y Levine, D.M. (1986). Concurrent and predictive validity of a selfreported measure of medication adherence. Medical Care, 24(1), 67-74.

Nishishinya, M.B., Rivera, J., Alegre, C., y Pereda, C.A. (2006). Intervenciones no farmacológicas y tratamientos alternativos en la fibromialgia. Medicina Clínica, 127(8), 295-299.

OMS (2004). Adherencia a los tratamientos a largo plazo. Pruebas para la acción. Génova: OMS.

Orueta, R. (2005). Estrategias para mejorar la adherencia terapéutica en patologías crónicas. Información Terapéutica Del Sistema Nacional De Salud, 29(2), 40-48.

Orueta, R. (2006). Evidencias disponibles para favorecer y mejorar la adherencia terapéutica. Revista Clínica de Medicina de Familia, 1(4), 185-192.

Pérez-Pareja, J., Borrás, C., Palmer, A., Sesé, A., Molina, F., y Gonzalvo, J. (2004). Fibromialgia y emociones negativas. Psicothema, 16(3), 415-420.

Redondo, J.R. (2011). Tratamiento farmacológico en la fibromialgia. Seminarios de la Fundación Española de Reumatología, 12(1), 21-26.

Sewitch, M.J., Dobkin, P.L., Bernatsky, S., Baron, M., Starr, M., Cohen, M., y Fitzcharles, M.A. (2004). Medication non-adherence in women with fibromyalgia. Rheumatology (Oxford, England), 43(5), 648-654. 
Ubago, M.C., Ruiz, I., Bermejo, M.J., Labry, A.O., y Plazaola, J. (2005). Clinical and psychosocial characteristics of subjects with fbromyalgia: Impact of the diagnosis on patients' activities. Revista Española de Salud Pública, 79(6), 683-695.

Van Dulmen, S., Sluijs, E., van Dijk, L., de Ridder, D., Heerdink, R., y Bensing, J. (2007). Patient adherence to medical treatment: A review of reviews. BMC Health Services Research, 7, 55. doi: 1472-6963-7-55 [pii].

Val, A., Amorós, G., Martínez, P., Fernández, M.L., y León, M. (1992). Estudio descriptivo del cumplimiento del tratamiento farmacológico antihipertensivo y validación del test de Morisky y Green. Atención Primaria, 10(5), 767-770.

Villanueva, V.L., Valía, J.C., Cerdá, G., Monsalve, V., Bayona, M.J., y Andrés, J.D. (2004). Fibromialgia: diagnóstico y tratamiento. El estado de la cuestión. Revista de la Sociedad Española del Dolor, 11(7), 50-63.

Recibido: 28 de junio de 2015 Recepción Modificaciones: 31 de julio de 2015 Aceptado: 3 de agosto de 2015 